\title{
The expression characteristics and prognostic roles of autophagy-related genes in gastric cancer
}

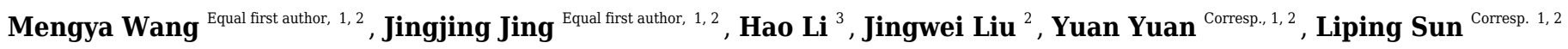 \\ ${ }^{1}$ Tumor Etiology and Screening Department of Cancer Institute, and Key Laboratory of Cancer Etiology and Prevention in Liaoning Education Department, \\ the First Hospital of China Medical University, Shenyang, China \\ 2 Key Laboratory of GI Cancer Etiology and Prevention in Liaoning Province, the First Hospital of China Medical University, Shenyang, China \\ 3 Department of Clinical Laboratory, the First Hospital of China Medical University, Shenyang, China \\ Corresponding Authors: Yuan Yuan, Liping Sun \\ Email address: yyuansubmission@hotmail.com, Ipsun@cmu.edu.cn
}

Background. Autophagy is an evolutionally highly conserved process, accompanied by the dynamic changes of various molecules, which is necessary for the orderly degradation and recycling of cellular components. The aim of the study was to identify the role of autophagy-related (ATG) genes in the occurrence and development of gastric cancer (GC). Methods. Data from Oncomine dataset was used for the differential expression analysis between cancer and normal tissues. The association of ATG genes expression with clinicopathologic indicators was evaluated by the Cancer Genome Atlas (TCGA) database and Gene Expression Omnibus (GEO) database. Moreover, using the TCGA datasets, the prognostic role of $A T G$ genes was assessed. A nomogram was further built to assess the independent prognostic factors. Results. The expression of autophagy-related genes AMBRA1, ATG4B, ATG7, ATG10, ATG12, ATG16L2, GABARAPL2, GABARAPL1, ULK4 and WIPI2 showed differences between cancer and normal tissues. After verification, ATG14 and ATG4D were significantly associated with TNM stage. ATG9A, ATG2A, and ATG4D were associated with T stage. VMP1 and ATG4A were low-expressed in patients without lymph node metastasis. No gene in autophagy pathway was associated with $\mathrm{M}$ stage. Further multivariate analysis suggested that ATG4D and MAP1LC3C were independent prognostic factors for GC. The C-index of nomogram was 0.676 and the $95 \% \mathrm{Cl}$ was 0.628 to 0.724 .

Conclusion. Our study provided a comprehensive illustration of ATG genes expression characteristics in GC. Abnormal expressions of the ubiquitin-like conjugated system in ATG genes plays a key role in the occurrence of GC. ATG8/LC3 sub-system may play an important role in development and clinical outcome of GC. In the future, it is necessary to further elucidate the alterations of specific ATG8/LC3 forms in order to provide insights for the discovery, diagnosis, or targeting for GC. 


\section{The Expression Characteristics and Prognostic Roles of}

\section{2 autophagy-related genes in gastric cancer}

3 Mengya Wang ${ }^{12 *}$, Jingjing Jing ${ }^{12 *}$, Hao Li ${ }^{3}$, Jingwei Liư ${ }^{2}$, Yuan Yuan ${ }^{12}$, Liping Sun ${ }^{12}$

$4{ }^{1}$ Tumor Etiology and Screening Department of Cancer Institute, and Key Laboratory of Cancer Etiology

5 and Prevention in Liaoning Education Department, the First Hospital of China Medical University,

6 Shenyang 110001, China

$7 \quad{ }^{2}$ Key Laboratory of GI Cancer Etiology and Prevention in Liaoning Province, the First Hospital of China

8 Medical University, Shenyang 110001, China

$9{ }^{3}$ Department of Clinical Laboratory, the First Hospital of China Medical University, Shenyang 110001,

10 China

11 * These authors contributed equally to this work and are considered to be co-first authors.

Corresponding Author:

Liping Sun ${ }^{12}$

No. 155, Nanjing North Street, Heping District, Shenyang City, Liaoning Province, 110001, China

17 Email address: Ipsun@cmu.edu.cn

Yuan Yuan ${ }^{12}$ 


\section{Abstract}

28 Background. Autophagy is an evolutionally highly conserved process, accompanied by the dynamic changes of various molecules, which is necessary for the orderly degradation and recycling of cellular components. The aim of the study was to identify the role of autophagyrelated $(A T G)$ genes in the occurrence and development of gastric cancer (GC).

Methods. Data from Oncomine dataset was used for the differential expression analysis between cancer and normal tissues. The association of $A T G$ genes expression with clinicopathologic indicators was evaluated by the Cancer Genome Atlas (TCGA) database and Gene Expression Omnibus (GEO) database. Moreover, using the TCGA datasets, the prognostic role of $A T G$ genes was assessed. A nomogram was further built to assess the independent prognostic factors. Results. The expression of autophagy-related genes $A M B R A 1, A T G 4 B, A T G 7, A T G 10, A T G 12$, ATG16L2, GABARAPL2, GABARAPL1, ULK4 and WIPI2 showed differences between cancer and normal tissues. After verification, $A T G 14$ and $A T G 4 D$ were significantly associated with TNM stage. $A T G 9 A, A T G 2 A$, and $A T G 4 D$ were associated with T stage. $V M P 1$ and $A T G 4 A$ were low-expressed in patients without lymph node metastasis. No gene in autophagy pathway was associated with $\mathrm{M}$ stage. Further multivariate analysis suggested that $A T G 4 D$ and $M A P 1 L C 3 C$ were independent prognostic factors for GC. The C-index of nomogram was 0.676 and the $95 \%$ CI was 0.628 to 0.724 .

Conclusion. Our study provided a comprehensive illustration of $A T G$ genes expression characteristics in GC. Abnormal expressions of the ubiquitin-like conjugated system in ATG genes plays a key role in the occurrence of GC. $A T G 8 / L C 3$ sub-system may play an important role in development and clinical outcome of GC. In the future, it is necessary to further elucidate the alterations of specific $A T G 8 / L C 3$ forms in order to provide insights for the discovery, diagnosis, or targeting for GC.

Key words: autophagy, gastric cancer, gene expression, TCGA, ATG4.

\section{Introduction}

Autophagy is an evolutionally highly conserved process, which is necessary for the orderly keeps low-level constitutive function. Basal autophagy plays an important role in maintaining homeostatic control and elimination of unfavorable proteins. Its activity can be accelerated by a variety of cellular stressors including nutrient starvation, DNA damage, and organelle damage. Autophagy is closely related to the occurrence and treatment of tumors (Rahman et al. 
61 have been widely observed. As a physiological quality control process, autophagy exerts a

62 cytoprotective effect to suppress cancer development by removing damage that leads to aberrant

63 mutations. On the other hand, as cancer progresses, starving and oxidative stress situation can

64 active autophagy to fulfill the high metabolic need of cancer cells (Mathew et al. 2007).

The process of autophagy is accompanied by the dynamic changes of various molecules.

66

67

68

69

70 Identification of the autophagy-related biomarkers will contribute to improving diagnosis and treatment of cancers. Autophagy is executed by a set of autophagy-related (ATG) genes, which have been investigated extensively in yeast. Although the discovery of $A T G$ genes greatly advanced the understanding of autophagy, the function and mechanisms involved in $A T G$ genes need to be further explored in mammalian. Recently, several studies have investigated the association of $A T G$ genes and cancers. By activating ATG6-mediated autophagy, the downregulation of microRNA-30a increases the chemoresistance of osteosarcoma cells, thereby inhibiting cell proliferation and invasion (Xu et al. 2016). Upregulation of UCA1 inhibits cell proliferation, migration, invasion, and drug resistance via ATG7-mediated autophagy (Wu et al. 2019). The methyltransferase MGMT inhibits the expression of ATG4B, thereby inhibiting autophagy and reducing the chemosensitivity of cisplatin in gastric cancer (GC) (Lei et al. 2020). Moreover, comprehensive study of all $A T G$ genes has been conducted in breast, head neck and kidney carcinoma (Deng et al. 2018; Pei et al. 2018).

$\mathrm{GC}$ is the fourth most common cancer and the second leading cause of cancer death in the world (Van Cutsem et al. 2016). The incidence is mainly related to diet, lifestyle, genetic predisposition, family history, treatment and medical conditions, infections, demographic characteristics, occupational exposures and ionizing radiation (Yusefi et al. 2018). Abnormal expression of $A T G$ genes may lead to the dysregulation of autophagy and tumorigenesis. However, the diagnostic and prognostic values of $A T G$ genes have not been fully realized in GC. Since largescale expression data is available, it is feasible to display an overview of $A T G$ genes from the perspective of expression characteristics and prognostic role in GC. In the current study, we performed systematic analysis by using available datasets of ONCOMINE and the Cancer Genome Atlas (TCGA), in order to evaluate the differential expression of $A T G$ genes and their associations with clinicopathological parameters and prognosis of GC. Our data may provide a new understanding of the autophagy-related mechanism in gastric carcinogenesis.

\section{Materials \& Methods}

\section{Autophagy-related genes selection}


94 Kyoto Encyclopedia of Genes and Genomes (KEGG, https://www.kegg.jp/) is an online tool for 95 analysis of the gene function (Kanehisa et al. 2020). Reactome (https://reactome.org/) is a 96 bioinformatics resource for visualization, interpretation and analysis of pathways (Jassal et al. 97 2020). Using the two datasets, we selected the genes in autophagy pathways as $A T G$ genes, 98 which composed four functional units including the ULK protein complex, Beclin-1/PI3K 99 complex, ubiquitin-like conjugation system and other genes (Mizushima et al. 2011). All the 100 isoforms of a gene were included, such as $A T G 4 A, A T G 4 B, A T G 4 C$ and $A T G 4 D$. A total of 40 101 genes were selected. The Pathvisio (Version:3.3.0, https:/www.pathvisio.org/) was used to 102 visualize the autophagy genes (Kutmon et al. 2015). which composed four functional units 103 including $U L K$ complex, $P I 3 K$ complex, ubiquitin-like conjugation system and other genes 104 (Table 1 and Fig. 1).

\section{Differential gene expression analysis by Oncomine}

106 By consulting the public data in Oncomine (https:/www.oncomine.org/resource/login.html)

107 (Rhodes et al. 2004), a powerful online database with 715 sub datasets and 86,733 samples, we

108 established and logged in an oncomine account (haoli@cmu.edu.cn), and input all of 40 ATG

109 genes (gene symbols were listed in Table 1) to analyze their differential expression at

110 transcription level in GC and different Lauren types. Combination of P-value $<0.05$ and fold

111 change $>2$ was identified as significant difference in gene expression.

112 Correlation analysis of $\boldsymbol{A T G}$ genes expression and clinicopathological parameters from 113 TCGA and GEO datasets

114 TCGA is a public database that contains the data of genomic expressions and the clinical features

115 in 33 types of cancers (Tomczak et al. 2015). The gene expression and clinicopathological

116 information of GC were downloaded from TCGA data portal

117 (https://portal.gdc.cancer.gov/projects/). R was performed to normalize the expression data. The

118 patients' TNM stage, T, N and M (Nagtegaal et al. 2020) were considered as the clinical

119 parameters.

120 Verification of the differences of gene expression

121 GSE62254 (https://www.ncbi.nlm.nih.gov/geo/query/acc.cgi?acc=GSE62254) was a 300

122 samples microarray profile tested by the Asian Cancer Research Group (ACRG) (Cristescu et al.

123 2015). Using GSE62254, we verified the differences of gene expression identified from TCGA

124 datasets.

\section{Statistical analysis}

126 All statistical analyses were performed by R 3.14 (http://www.r-project.org/) and the package of 127 rms. Student's $t$-tests was used to analyze the differences between cancer samples and normal 
128 tissues, of which the criterion is $\mathrm{p}$-value $<0.01$ and fold change $>2.0$. The association between

129 the $A T G$ genes expressions and clinical features was accessed by Pearson $\mathrm{X}^{2}$ test. The correlation

130 between $A T G$ genes expressions and overall survival time was evaluated by Kaplan-Meier

131 method and compared by log-rank test. Univariate and multivariate Cox proportional hazard

132 regression models were used to recognize the independent prognostic factors. Based on the

133 multivariate Cox regression models, a nomogram was formulated together with all the

134 independent prognostic genes. The concordance index (C-index), which is similar to the area

135 under the receiver operating characteristic (ROC), was used to evaluate the nomogram. $\mathrm{P}<0.05$

136 were considered significant difference.

137

\section{Results}

\section{Differential expression of $\boldsymbol{A T G}$ genes in GC}

140 By the Oncomine analysis, there were 10 genes of $40 A T G$ genes with significantly differential

141 expression between GC and normal samples, which were named as differentially expressed

142 genes (DEGs) (Fig. 2). Seven DEGs were belong to the ubiquitin-like conjugating system,

143 among them $A T G 4 B, A T G 12$ and $A T G 16 L 2$ were significantly up-regulated in GC, while

144 ATG10, GABARAPL2 and GABARAPL1 expressions were down-regulated in GC. As for ATG7,

145 the expression was uncertain. $U L K 4$, belonging to the $U L K$ complex, was found down-regulated

146 in GC. While $A M B R A 1$, a member of the $P I 3 K$ complex, was highly expressed in GC. As a

147 connection between PI3K complex, ULK complex and ATG12 system, WIPI2 showed higher

148 expression in cancer tissue.

149 Histological stratification analysis showed that GABARAPL1 was down-regulated in all

150 types of GC compared with normal tissues, with fold change of -2.321 in intestinal gastric

151 adenocarcinoma, -2.287 fold in diffuse adenocarcinoma and -2.622 fold in mixed

152 adenocarcinoma (Cho Gastric, Cho,J.Y. 2011). Six DEGs showed significant differences in the

153 gastric mixed adenocarcinoma subgroup, among them $A M B R A 1, A T G 4 B, A T G 7$ (probe

154 224025_s_at) and ATG12 were up-regulated, while GABARAPL1 and ATG7 (probe 1569827_at)

155 were down-regulated. Four DEGs including ATG10, ATG16L2, ULK4 and GABARAPL1 showed

156 differences in diffuse gastric adenocarcinoma subgroup, while other four DEGs including ATG7

157 (probe 224025_s_at), GABARAPL1, WIPI2 and GABARAPL3 showed differences in gastric

158 intestinal type adenocarcinoma subgroup (DErrico Gastric, D'Errico, M. 2009) (Fig. 3A and 3B).

159 Association between $\boldsymbol{A T G}$ genes expression and clinicopathologic variables of GC

160 Data of 376 GC patients in TCGA were downloaded for the analysis. ATG14, ULK3, ATG2B,

$161 A T G 12, A T G 4 C, A T G 4 D$, and $M A P 1 L C 3 A$ showed significantly relationship with TNM stage. 
162 After verification, $A T G 14$ and $A T G 4 D$ were significantly associated with TNM stage $(\mathrm{P}=0.027$,

1630.048 respectively). ATG9A $(\mathrm{P}=0.00083), A T G 2 A(\mathrm{P}=0.00417)$, and $A T G 4 D(\mathrm{P}=0.00864)$ were

164 related with T stage. Low expression of $V M P 1$ and $A T G 4 A$ suggested absence of lymph node

165 metastasis ( $\mathrm{P}=0.0018,0.015$, correspondingly). However, no gene in autophagy pathway was

166 observed to be associated with M stage (Table 2).

\section{Roles of $\boldsymbol{A T G}$ genes expression in the prediction of GC prognosis}

168354 patients were included to analyze the overall survival of GC. The median value was used as

169 cut-off value to distinguish high expression and low expression of $A T G$ genes. According to the

170 univariate survival analysis, $A T G 4 D, G A B A R A P L 2$ and $M A P 1 L C 3 C$ were significantly

171 associated with the prognosis of GC. Moreover, the patients with low-expression of $A T G 4 D$ or

172 high-expression of GABARAPL2 and MAP1LC3C demonstrated longer survival time, and both

173 of the latter two genes belonged to $A T G 8 / L C 3$ system. Using the Cox's proportional hazards

174 model, we then performed the multivariate analysis adjusted by gender, age, TNM stage. ATG4D

175 and MAPILC3C were identified as the independent prognostic factors, with adjusted hazard ratio

176 (HR) of 1.5727 (95\%CI 1.1194-2.21) and 0.5767 (95\%CI 0.4086-0.8138) separately (Fig. 4 and

177 Table 3). The summary of the correlation between ATG genes expression and TNM staging and

178 prognosis of GC was shown in Fig. 5.

179 Joint prediction of the GC prognosis using $A T G 4 D$ and $M A P 1 L C 3 C$

180 According to the expression of $A T G 4 D$ and MAP1LC3C in GC, the gastric cancer patients were

181 divided into four groups: $A T G 4 D$ high expression - MAP1LC $3 C$ high expression (HH), ATG4D

182 low expression - MAP $1 L C 3 C$ low expression (LL), ATG4D high expression - MAP $1 L C 3 C$ low

183 expression (HL) and $A T G 4 D$ low expression - MAP1LC3C high expression (LH). A significant

184 difference was displayed among the four groups ( $\mathrm{p}=0.0056$, Fig. 6A).

185 Furtherly, to predict 1-year and 3-year survival rate, we built a nomogram by the

186 multivariate Cox regression models. After validation, the $\mathrm{C}$-index was 0.676 and the $95 \% \mathrm{CI}$ was

1870.628 to 0.724 . According to the total score after added with points identified on the point scale,

188 we found that the likelihood of 1-year and 3-year OS for individual patient could be reasonably

189 predicated by nomogram (Fig. 6B). As shown in Fig. 6C and 6D, the survival evaluated by the

190 Kaplan-Meier method was marked on the y-axes, the predicted survival estimated by nomogram

191 was observed on the x-axes, and the red lines represented the ideal reference line for which

192 predicted survival corresponds with actual survival. The plot for the probability of OS 1-year or

193 3-year showed optimal agreement between the prediction by nomogram and actual observation

194 for nomogram.

195 


\section{Discussion}

197 Considering the vital function of $A T G$ genes in autophagy, many studies have focused on

198 their role in cancers. To date, no researcher has elaborated an overview of the impact of $A T G$

199 genes on the development, progression, and prognosis of GC. In the current study, for the first

200 time, we investigated the expression profiling and the prognostic roles of whole members of

$201 A T G$ genes in GC using multiple databases. Our results elucidated that abnormal expressions of

202 some key $A T G$ genes were significantly associated with GC progression and outcome.

203

Firstly, 10 DEGs were identified between cancer and normal tissues, and 7 of these genes

204 were associated with ubiquitin-like conjugating system, which intimately involved in driving the biogenesis of the autophagosomal membrane (Nakatogawa 2013). ATG4B (Liu et al. 2014), the core autophagy protein in $A T G 8 / L C 3$ system, was found to be up-regulated in cancer tissue in our study. It has been reported that $A T G 4 B$ can promote the growth of colorectal cancer, while silencing the expression of $A T G 4 B$ can reduce the colony formation of cancer cells and inhibit tumor growth (Liu et al. 2014; PF et al. 2018). The E1-like activating enzyme ATG7 and the E2like activating enzyme $A T G 10$ also play a vital role in activating and transferring key proteins in the sub-systems. In our study, expression of $A T G 12$ and $A T G 7$ showed up-regulation while ATG10 expression was down regulated in cancer tissues. Similarly, Cao, Q. H et al (Cao et al. 2016) analyzed 352 tissue microarrays containing cancer and paired adjacent normal tissues and found that $A T G 7, A T G 12$ were highly expressed in the GC tissues, and $A T G 10$ was weakly tumor promoter, mainly depends on the environment, and its activity will change with the development of the tumor (Amirfallah et al. 2019). As for $A T G$ genes of other functional units of ubiquitin-like conjugating system, some studies (Lebovitz et al. 2015; Su et al. 2019) found that GABARAPL1 transcripts were less abundant in breast, prostate, liver and non-small cell lung cancers than matched normal controls, indicating that GABARAPL1 may be a tumor suppressor. While ATG16L2 transcripts increased in kidney cancer. As a high risk gene, its high expression is associated with poor prognosis (Wan et al. 2019). The high expression of Ambra1 is beneficial to cell survival (Sun et al. 2018). Falasca compared 26 prostate adenocarcinoma and 12 normal specimens by immunohistochemistry and observed that $A M B R A 1$ was highly expressed in prostate cancer (Falasca et al. 2015). The expression trend of those genes was consistent with our results in GC. The above results indicate that the ubiquitin-like conjugated system plays a key role in the occurrence of GC, and its mechanism deserves further study. 
230 genes and GC TNM staging, and verified the differential genes using GSE62254 to improve the 231 accuracy. After verification, $A T G 9 A, A T G 2 A$ and $A T G 4 D$ were found to be associated with T

232 stage. Among these genes, $A T G 9 A$ was previously reported to be associated with T stage in non233 metastatic renal cell carcinoma (Tang et al. 2013). ATG4D affects the biological behavior of 234 prostate cancer by regulating the activity of androgen receptor ( $\mathrm{Hu}$ et al. 2020). Besides, all of 235 these significant differences were observed at early $\mathrm{T}$ stage, which suggested that autophagy may 236 play its role mainly at the early stage of GC progression. By analyzing the expression of $A T G$ 237 genes both in TCGA and GSE62254, the results showed that VMP1 and ATG4A were over238 expressed in patients with lymph node metastasis. Similarly, Yang et al found that the expression 239 of $A T G 4 A$ was associated with lymph node metastasis in $110 \mathrm{GC}$ patients (Yang et al. 2016).

$240 V M P 1$ was reported to promote Kras G12D-mediated pancreatic cancer initiation and facilitate 241 lymph node metastasis (Loncle et al. 2016). In addition, $A T G 4 D$ and $A T G 14$ were observed to be 242 associated with overall TNM stage according to our analysis. ATG14 was up-regulated while $243 A T G 4 D$ was down-regulated in GC of stage III-IV, which suggested that the two genes may have 244 the opposite effect in GC progression. It has been reported that the low expression of $A T G 4 D$ 245 was associated with Colorectal Cancer of III stage (Gil et al. 2018). Interestingly, significant 246 relation was observed between $A T G 4$ isoforms and all the three clinicopathologic variables, that $247 A T G 4 D$ was associated with TNM and T stage, and $A T G 4 A$ showed difference in N stage. As 248 ATG4 activity is essential and highly specific to autophagy, it may be a prospective autophagy249 specific target for GC therapy.

250 Previous investigations have also indicated the predictive role of autophagy pathway genes 251 in prognosis of various types of cancers. Here we analyzed all the $A T G$ genes using TCGA 252 dataset to assess their prognostic values in GC. $A T G 4 D$ and MAP1LC $3 C$ were confirmed to be 253 statistically significant in multivariate survival analysis. The expression of $A T G 4 D$ and $254 M A P 1 L C 3 C$ is low in colorectal cancer, and $A T G 4 D$ is related to the poor prognosis of 255 pancreatic cancer ( $\mathrm{Hu}$ et al. 2020). The high expression of $A T G 4 D$ and the low expression of $256 M A P 1 L C 3 C$ may indicate the poor survival of gastric patients. Furthermore, we developed a 257 nomogram according to the joint expression of $A T G 4 D$ and MAP $1 L C 3 C$ along with other 258 clinicopathological parameters. The group of HL showed poor survival while the group of LH 259 indicated favorable prognosis. In the internal validation set, the calibration plot showed that the 260 predicted 3-year and 5-year overall survival were in correspondence with the actual survival 261 estimated by the Kaplan-Meier method. MAP1LC3C is a member of the LC3 family of proteins 262 and a key structural component of the autophagosome that undergoes processing by members of 263 the ATG4 family (Costa et al. 2016). These two functionally related genes together may have 
264 synergistic effect in GC prognosis. For the first time, our study formulated an $A T G$-based

265

266

267

268

269

270

271

272

273

274

275

276

277

278

279

280

281

282

283

284

285

286

287

288

289

290

291

292

293

294

295

296

297

nomogram that could predict outcome of GC with a better accuracy.

On the basis of the above results, we found that $A T G 4$ and $A T G 8$, members of $A T G 8 / L C 3$ system, were associated with both the occurrence and prognosis of GC in our study. ATG4 was up-regulated in cancer and was associated with poor GC survival. The over-expression of $A T G 8$ was observed in normal tissues and involved with favorable prognosis of GC. ATG8/LC3 is essential for autophagosome biogenesis and it also functions as an adaptor protein for selective autophagy (Lee \& Lee 2016). At the same time, it is also widely used as a marker of autophagic vacuoles (OA et al. 2020). Dysregulation of $A T G 8 / L C 3$ proteins may contribute to pathogenic effects during progression of autophagy-associated human diseases. Our results indicated that the $A T G 8 / L C 3$ system may play an important role in development and clinical outcome of GC. Elucidation of alterations in specific $A T G 8 / L C 3$ forms in GC could provide insights for the discovery, diagnosis, or targeting of this high-mortality disease.

In conclusion, our study provided a comprehensive illustration of $A T G$ genes expression characteristics in GC. Abnormal expressions of $A T G$ genes were observed to be significantly involved in the whole process of GC occurrence, progression and prognosis. Specially, the $U L K$ system, such as $A T G 4$ family and $A T G 8 / L C 3$, may serve as valuable biomarkers to indicate gastric carcinogenesis and prognosis. Considering the underlying important roles of $A T G$ genes in gastric carcinogenesis and progression, future molecular experiments concerning the functions and mechanisms of $A T G$ genes may generate promising significance in GC development and treatment.

\section{Conclusions}

Our study provided a comprehensive illustration of $A T G$ genes expression characteristics in GC. Abnormal expressions of the ubiquitin-like conjugated system in $A T G$ genes plays a key role in the occurrence of GC. $A T G 8 / L C 3$ sub-system may play an important role in development and clinical outcome of GC. In the future, it is necessary to further elucidate the alterations of specific $A T G 8 / L C 3$ forms in order to provide insights for the discovery, diagnosis, or targeting for GC.

\section{References}

Amirfallah A, Arason A, Einarsson H, Gudmundsdottir ET, Freysteinsdottir ES, Olafsdottir KA, Johannsson OT, Agnarsson BA, Barkardottir RB, and Reynisdottir I. 2019. High expression of the vacuole membrane protein 1 (VMP1) is a potential marker of poor 
298

299

300

301

302

303

304

305

306

307

308

309

310

311

312

313

314

315

316

317

318

319

320

321

322

323

324

325

326

327

328

329

330

331

332

333

334

335

prognosis in HER2 positive breast cancer. PLoS One 14:e0221413.

10.1371/journal.pone.0221413

Cao QH, Liu F, Yang ZL, Fu XH, Yang ZH, Liu Q, Wang L, Wan XB, and Fan XJ. 2016. Prognostic value of autophagy related proteins ULK1, Beclin 1, ATG3, ATG5, ATG7, ATG9, ATG10, ATG12, LC3B and p62/SQSTM1 in gastric cancer. Am J Transl Res 8:3831-3847.

Costa JR, Prak K, Aldous S, Gewinner CA, and Ketteler R. 2016. Autophagy gene expression profiling identifies a defective microtubule-associated protein light chain 3A mutant in cancer. Oncotarget 7:41203-41216. 10.18632/oncotarget.9754

Cristescu R, Lee J, Nebozhyn M, Kim KM, Ting JC, Wong SS, Liu J, Yue YG, Wang J, Yu K, Ye XS, Do IG, Liu S, Gong L, Fu J, Jin JG, Choi MG, Sohn TS, Lee JH, Bae JM, Kim ST, Park SH, Sohn I, Jung SH, Tan P, Chen R, Hardwick J, Kang WK, Ayers M, Hongyue D, Reinhard C, Loboda A, Kim S, and Aggarwal A. 2015. Molecular analysis of gastric cancer identifies subtypes associated with distinct clinical outcomes. Nat Med 21:449-456. 10.1038/nm.3850

Deng Q, Liang L, Liu Q, Duan W, Jiang Y, and Zhang L. 2018. Autophagy is a major mechanism for the dual effects of curcumin on renal cell carcinoma cells. Eur $J$ Pharmacol 826:24-30. 10.1016/j.ejphar.2018.02.038

Falasca L, Torino F, Marconi M, Costantini M, Pompeo V, Sentinelli S, De Salvo L, Patrizio M, Padula C, Gallucci M, Piacentini M, and Malorni W. 2015. AMBRA1 and SQSTM1 expression pattern in prostate cancer. Apoptosis 20:1577-1586. 10.1007/s10495-0151176-3

Gil J, Ramsey D, Pawlowski P, Szmida E, Leszczynski P, Bebenek M, and Sasiadek MM. 2018. The Influence of Tumor Microenvironment on ATG4D Gene Expression in Colorectal Cancer Patients. Med Oncol 35:159. 10.1007/s12032-018-1220-6

Hu D, Jiang L, Luo S, Zhao X, Hu H, Zhao G, and Tang W. 2020. Development of an autophagy-related gene expression signature for prognosis prediction in prostate cancer patients. J Transl Med 18:160. 10.1186/s12967-020-02323-x

Jassal B, Matthews L, Viteri G, Gong C, Lorente P, Fabregat A, Sidiropoulos K, Cook J, Gillespie M, Haw R, Loney F, May B, Milacic M, Rothfels K, Sevilla C, Shamovsky V, Shorser S, Varusai T, Weiser J, Wu G, Stein L, Hermjakob H, and D'Eustachio P. 2020. The reactome pathway knowledgebase. Nucleic Acids Res 48:D498-d503. 10.1093/nar/gkz1031

Kanehisa M, Furumichi M, Sato Y, Ishiguro-Watanabe M, and Tanabe M. 2020. KEGG: integrating viruses and cellular organisms. Nucleic Acids Res. 10.1093/nar/gkaa970

Kutmon M, van Iersel MP, Bohler A, Kelder T, Nunes N, Pico AR, and Evelo CT. 2015. PathVisio 3: an extendable pathway analysis toolbox. PLoS Comput Biol 11:e1004085. 10.1371/journal.pcbi.1004085 
336 Lebovitz CB, Robertson AG, Goya R, Jones SJ, Morin RD, Marra MA, and Gorski SM. 2015.

337

338

339

340

341

342

343

344

345

346

347

348

349

350

351

352

353

354

355

356

357

358

359

360

361

362

363

364

365

366

367

368

369

370

371

372

373

Cross-cancer profiling of molecular alterations within the human autophagy interaction network. Autophagy 11:1668-1687. 10.1080/15548627.2015.1067362

Lee YK, and Lee JA. 2016. Role of the mammalian ATG8/LC3 family in autophagy: differential and compensatory roles in the spatiotemporal regulation of autophagy. BMB Rep 49:424430.

Lei Y, Tang L, Hu J, Wang S, Liu Y, Yang M, Zhang J, and Tang B. 2020. Inhibition of MGMT-mediated autophagy suppression decreases cisplatin chemosensitivity in gastric cancer. Biomed Pharmacother 125:109896. 10.1016/j.biopha.2020.109896

Liu PF, Leung CM, Chang YH, Cheng JS, Chen JJ, Weng CJ, Tsai KW, Hsu CJ, Liu YC, Hsu PC, Pan HW, and Shu CW. 2014. ATG4B promotes colorectal cancer growth independent of autophagic flux. Autophagy 10:1454-1465. 10.4161/auto.29556

Loncle C, Molejon MI, Lac S, Tellechea JI, Lomberk G, Gramatica L, Fernandez Zapico MF, Dusetti N, Urrutia R, and Iovanna JL. 2016. The pancreatitis-associated protein VMP1, a key regulator of inducible autophagy, promotes Kras(G12D)-mediated pancreatic cancer initiation. Cell Death Dis 7:e2295. 10.1038/cddis.2016.202

Mathew R, Karantza-Wadsworth V, and White E. 2007. Role of autophagy in cancer. Nat Rev Cancer 7:961-967. 10.1038/nrc2254

Mizushima N, Yoshimori T, and Ohsumi Y. 2011. The role of Atg proteins in autophagosome formation. Annu Rev Cell Dev Biol 27:107-132. 10.1146/annurev-cellbio-092910-154005

Nagtegaal ID, Odze RD, Klimstra D, Paradis V, Rugge M, Schirmacher P, Washington KM, Carneiro F, and Cree IA. 2020. The 2019 WHO classification of tumours of the digestive system. Histopathology 76:182-188. 10.1111/his.13975

Nakatogawa H. 2013. Two ubiquitin-like conjugation systems that mediate membrane formation during autophagy. Essays Biochem 55:39-50. 10.1042/bse0550039

OA M, W J, SR G, CL H, DDH T, M P, DL D, AS G, I G, and Autophagy GGJ. 2020. Transgenic expression of GFP-LC3 perturbs autophagy in exocrine pancreas and acute pancreatitis responses in mice.1-14. 10.1080/15548627.2020.1715047

Pei L, Kong Y, Shao C, Yue X, Wang Z, and Zhang N. 2018. Heme oxygenase-1 induction mediates chemoresistance of breast cancer cells to pharmorubicin by promoting autophagy via PI3K/Akt pathway. J Cell Mol Med. 10.1111/jcmm.13800

PF L, KL T, CJ H, WL T, JS C, HW C, CW S, YG G, HH T, CH W, JC R, LW Y, and Theranostics SCJ. 2018. Drug Repurposing Screening Identifies Tioconazole as an ATG4 Inhibitor that Suppresses Autophagy and Sensitizes Cancer Cells to Chemotherapy. 8:830-845. 10.7150/thno.22012

Rahman MA, Saha SK, Rahman MS, Uddin MJ, Uddin MS, Pang MG, Rhim H, and Cho SG. 2020. Molecular Insights Into Therapeutic Potential of Autophagy Modulation by Natural Products for Cancer Stem Cells. Front Cell Dev Biol 8:283. 10.3389/fcell.2020.00283

Peer) reviewing PDF | (2020:09:52988:1:3:NEW 2 Dec 2020) 
374 Rhodes DR, Yu J, Shanker K, Deshpande N, Varambally R, Ghosh D, Barrette T, Pandey A, and

375

376

377

378

379

380

381

382

383

384

385

386

387

388

389

390

391

392

393

394

395

396

397

398

399

400

401

402

403

404

405

406

407

408

409 Chinnaiyan AM. 2004. ONCOMINE: a cancer microarray database and integrated datamining platform. Neoplasia 6:1-6.

Su B, Zhang L, Liu S, Chen X, and Zhang W. 2019. GABARAPL1 Promotes AR+ Prostate Cancer Growth by Increasing FL-AR/AR-V Transcription Activity and Nuclear Translocation. Front Oncol 9:1254. 10.3389/fonc.2019.01254

Sun WL, Wang L, Luo J, Zhu HW, and Cai ZW. 2018. Ambral modulates the sensitivity of breast cancer cells to epirubicin by regulating autophagy via ATG12. Cancer Sci 109:3129-3138. 10.1111/cas.13743

Tang JY, Hsi E, Huang YC, Hsu NC, Chen YK, Chu PY, and Chai CY. 2013. ATG9A overexpression is associated with disease recurrence and poor survival in patients with oral squamous cell carcinoma. Virchows Arch 463:737-742. 10.1007/s00428-013-1482-5

Tomczak K, Czerwinska P, and Wiznerowicz M. 2015. The Cancer Genome Atlas (TCGA): an immeasurable source of knowledge. Contemp Oncol (Pozn) 19:A68-77. $10.5114 /$ wo.2014.47136

Van Cutsem E, Sagaert X, Topal B, Haustermans K, and Prenen H. 2016. Gastric cancer. Lancet 388:2654-2664. 10.1016/s0140-6736(16)30354-3

Wan B, Liu B, Yu G, Huang Y, and Lv C. 2019. Differentially expressed autophagy-related genes are potential prognostic and diagnostic biomarkers in clear-cell renal cell carcinoma. Aging (Albany NY) 11:9025-9042. 10.18632/aging.102368

Wu J, Li W, Ning J, Yu W, Rao T, and Cheng F. 2019. Long noncoding RNA UCA1 targets miR-582-5p and contributes to the progression and drug resistance of bladder cancer cells through ATG7-mediated autophagy inhibition. Onco Targets Ther 12:495-508. $10.2147 /$ ott.S183940

Xu R, Liu S, Chen H, and Lao L. 2016. MicroRNA-30a downregulation contributes to chemoresistance of osteosarcoma cells through activating Beclin-1-mediated autophagy. Oncol Rep 35:1757-1763. 10.3892/or.2015.4497

Yang SW, Ping YF, Jiang YX, Luo X, Zhang X, Bian XW, and Yu PW. 2016. ATG4A promotes tumor metastasis by inducing the epithelial-mesenchymal transition and stem-like properties in gastric cells. Oncotarget 7:39279-39292. 10.18632/oncotarget.9827

Yang Y, Li X, Wang T, Guo Q, Xi T, and Zheng L. 2020. Emerging agents that target signaling pathways in cancer stem cells. J Hematol Oncol 13:60. 10.1186/s13045-020-00901-6

Yusefi AR, Bagheri Lankarani K, Bastani P, Radinmanesh M, and Kavosi Z. 2018. Risk Factors for Gastric Cancer: A Systematic Review. Asian Pac J Cancer Prev 19:591-603. 10.22034/apjcp.2018.19.3.591 
Figure 1

\section{Schematic of autophagy pathway.}

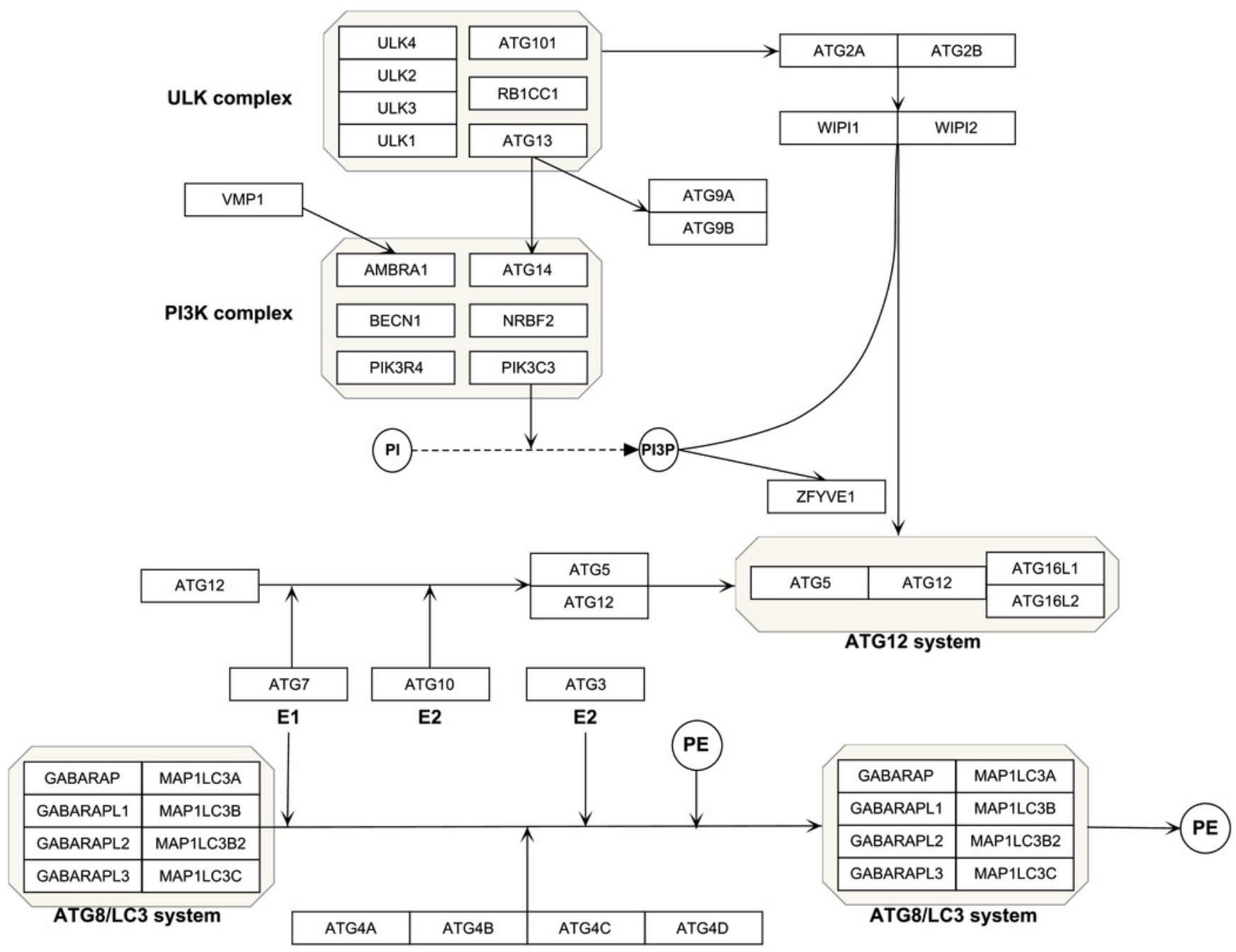


Figure 2

Different ATGs mRNA expression in different tumor types.

This graphic showed the numbers of datasets with statistically signifcant mRNA

overexpression (red) or downexpression (blue) of the target gene (cancer vs. normal tissue).

Cell color is determined by the best gene rank percentile for the analyses within the cell.

\begin{tabular}{|r|}
\hline Analysis Type by Cancer \\
\hline Bladder cancer \\
\hline Brain and CNS cancer \\
\hline Breast cancer \\
\hline Cervival cancer \\
\hline Colorectal cancer \\
\hline Esophageal cancer \\
\hline Gastric cancer \\
\hline Head and Neck cancer \\
\hline Kidney cancer \\
\hline Leukemia \\
\hline Liver cancer \\
\hline Lung cancer \\
\hline Lymphoma \\
\hline Melanoma \\
\hline Myeloma \\
\hline Other cancer \\
\hline Ovarian cancer \\
\hline Pancreatic cancer \\
\hline Prostate cancer \\
\hline Sarcoma \\
\hline Total Unique Analyses \\
\hline
\end{tabular}

\begin{tabular}{|c|c|c|c|c|c|c|c|c|c|c|c|c|c|c|c|c|c|c|c|}
\hline \multirow{2}{*}{\multicolumn{2}{|c|}{$\begin{array}{c}\text { AMBRA1 } \\
\text { Cancer } \\
\text { vs. } \\
\text { Normal }\end{array}$}} & \multirow{2}{*}{\multicolumn{2}{|c|}{$\begin{array}{c}\text { ATG4B } \\
\text { Cancer } \\
\text { vs. } \\
\text { Normal }\end{array}$}} & \multicolumn{2}{|c|}{ ATG7 } & \multicolumn{2}{|c|}{ ATG10 } & \multicolumn{2}{|c|}{ ATG12 } & \multicolumn{2}{|c|}{ ATG16L2 } & \multicolumn{2}{|c|}{ GABARAPL2 } & \multicolumn{2}{|c|}{ GABARAPL1 } & \multicolumn{2}{|c|}{ ULK4 } & \multicolumn{2}{|c|}{ WIPI2 } \\
\hline & & & & \multicolumn{2}{|c|}{$\begin{array}{l}\text { Cancer } \\
\text { vs. } \\
\text { Normal }\end{array}$} & \multicolumn{2}{|c|}{$\begin{array}{l}\text { Cancer } \\
\text { vs. } \\
\text { Normal }\end{array}$} & \multicolumn{2}{|c|}{$\begin{array}{l}\text { Cancer } \\
\text { vs. } \\
\text { Normal }\end{array}$} & \multicolumn{2}{|c|}{$\begin{array}{l}\text { Cancer } \\
\text { vs. } \\
\text { Normal }\end{array}$} & \multicolumn{2}{|c|}{$\begin{array}{l}\text { Cancer } \\
\text { vs. } \\
\text { Normal }\end{array}$} & \multicolumn{2}{|c|}{$\begin{array}{l}\text { Cancer } \\
\text { vs. } \\
\text { Normal }\end{array}$} & \multicolumn{2}{|c|}{$\begin{array}{l}\text { Cancer } \\
\text { vs. } \\
\text { Normal }\end{array}$} & \multicolumn{2}{|c|}{$\begin{array}{l}\text { Cancer } \\
\text { vs. } \\
\text { Normal }\end{array}$} \\
\hline & & & & & & & & 1 & & & & & & & 2 & & & 1 & \\
\hline & 1 & & 3 & & & & 1 & & 1 & 2 & & & 10 & & 6 & 3 & 2 & & \\
\hline \multirow[t]{4}{*}{1} & & & 1 & & & & 1 & & 1 & 1 & & & 23 & & 8 & & 1 & 1 & \\
\hline & & & & & & & & & & & & & & & & & & & \\
\hline & 1 & & & 2 & & & & & & & & & 6 & & 6 & 4 & & & \\
\hline & 1 & & & & & 1 & & & & & & & & & & & & & \\
\hline \multirow[t]{2}{*}{1} & & 1 & & 2 & 1 & & 1 & 1 & & 1 & & & 4 & & 1 & & 1 & 2 & 1 \\
\hline & & & & & & & & 3 & 1 & & & & 2 & & - & & & & \\
\hline \multirow[t]{4}{*}{6} & & & & & & & & 2 & & & & 1 & 7 & 1 & 4 & 2 & 1 & 2 & \\
\hline & & 1 & 1 & & 3 & & & & 2 & & 4 & 2 & 7 & 1 & 5 & & 1 & & \\
\hline & & & 1 & & & & & & & & & 1 & 2 & 1 & 2 & & 1 & & \\
\hline & & & & & & & & 2 & & & 1 & & 9 & 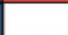 & 2 & & & & 1 \\
\hline \multirow[t]{2}{*}{1} & & 1 & & 3 & & 1 & & 1 & & & & 2 & 3 & 5 & 4 & 1 & & 3 & \\
\hline & & & & & & & & & & & 2 & & & & 2 & & & 1 & \\
\hline \multirow[t]{6}{*}{1} & & & & 1 & & & & 1 & & & & & & 2 & & & & 1 & \\
\hline & & 1 & 1 & & & & & 2 & & 1 & 2 & 7 & 2 & 4 & 5 & & 6 & 1 & 7 \\
\hline & & & & & & & 1 & & & & & & 5 & & 1 & 1 & & & \\
\hline & & 1 & & & & & & & & & & 2 & 1 & & & & & & \\
\hline & & & 2 & & & & & & & & & & & & & & & & \\
\hline & & & & & & & & 7 & & & & & 9 & & 11 & & & & 1 \\
\hline 11 & 3 & 5 & 9 & 8 & 4 & 2 & 4 & 21 & 5 & 5 & 9 & 15 & 90 & 14 & 57 & 11 & 13 & 12 & 10 \\
\hline \multicolumn{2}{|c|}{359} & \multicolumn{2}{|c|}{391} & \multicolumn{2}{|c|}{374} & \multicolumn{2}{|c|}{287} & & & & & & 25 & 2 & & 33 & & 41 & 2 \\
\hline & & & & & & 1 & & & & & 0 & 5 & 1 & & & & & & \\
\hline
\end{tabular}


Figure 3

The detail information for the different ATGs.

(A)The detail information in the oncomine dataset. (B)The position of different ATGs in autophagy pathway. The blue color represents downexpression in cancer, while the red color represents overexpression in cancer. The gene with two different colors means two probe of the gene showed different expressions. The gradient color represents the gene's fold change.

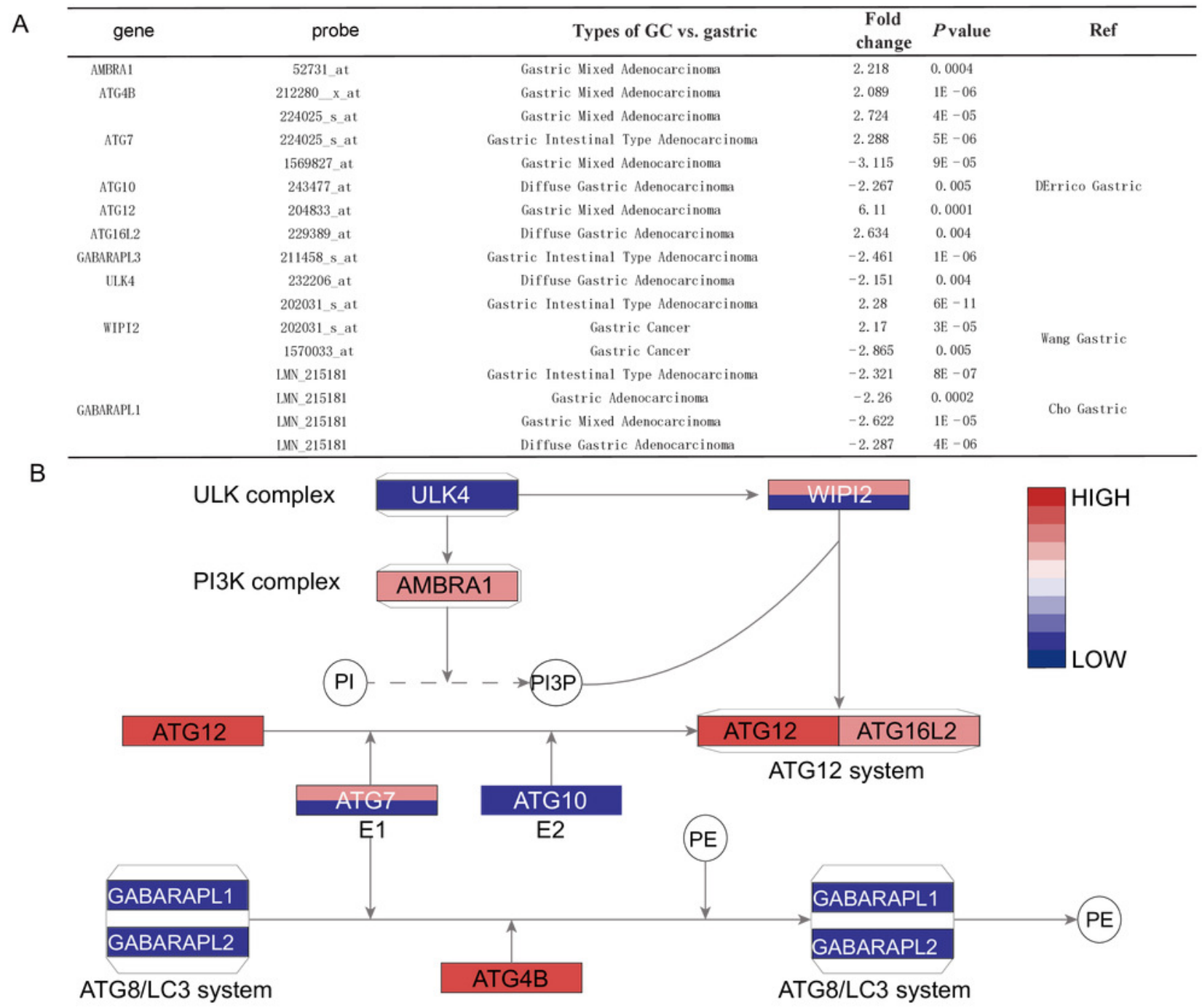


Figure 4

The prognostic value of mRNA level of independent prognostic factors.

(A)ATG4D. (B)MAP1LC3C.

A

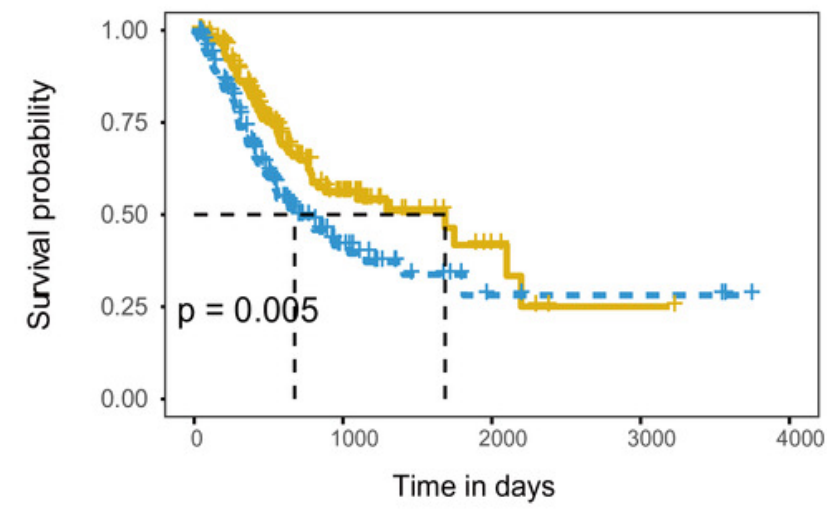

B $\quad$ MAP1LC3C - low-expression + high-expression

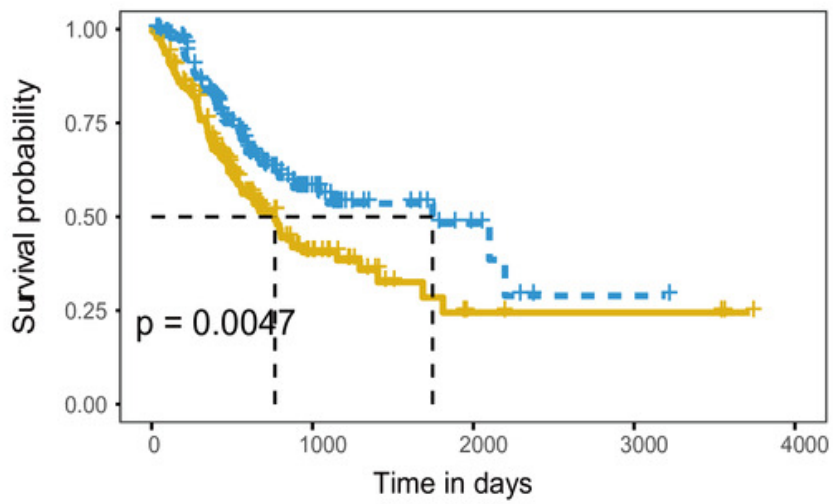

Number at risk

\begin{tabular}{|c|c|c|c|c|c|c|c|c|c|c|c|}
\hline \multicolumn{4}{|c|}{ Number at risk } & \multicolumn{8}{|c|}{ Number at risk } \\
\hline $\begin{array}{l}\text { low-expression } \\
\text { high-expressior }\end{array}$ & \begin{tabular}{|l|}
177 \\
177 \\
\end{tabular} & $\begin{array}{l}34 \\
22 \\
\end{array}$ & $\begin{array}{l}6 \\
4 \\
\end{array}$ & $\begin{array}{l}1 \\
3\end{array}$ & $\begin{array}{l}0 \\
0\end{array}$ & low-expression & $\begin{array}{l}177 \\
177\end{array}$ & $\begin{array}{l}23 \\
33 \\
\end{array}$ & $\begin{array}{l}4 \\
6\end{array}$ & $\begin{array}{l}3 \\
1\end{array}$ & $\begin{array}{l}0 \\
0\end{array}$ \\
\hline & 0 & 1000 & 2000 & 3000 & 4000 & & 0 & 1000 & 2000 & 3000 & 4000 \\
\hline & & & in $d$ & & & & & & in da & & \\
\hline
\end{tabular}




\section{Figure 5}

Summary of the correlation between ATG genes expression and TNM staging and prognosis.

The red frame represents genes with high significant expression, and the blue frame represents genes with low significant expression. "**" represents that gene expression has significant correlation with TNM staging or OS of GC.

High gene expression in Oncomine

Low gene expression in Oncomine $\quad$ ** $\mathrm{P}<0.05$

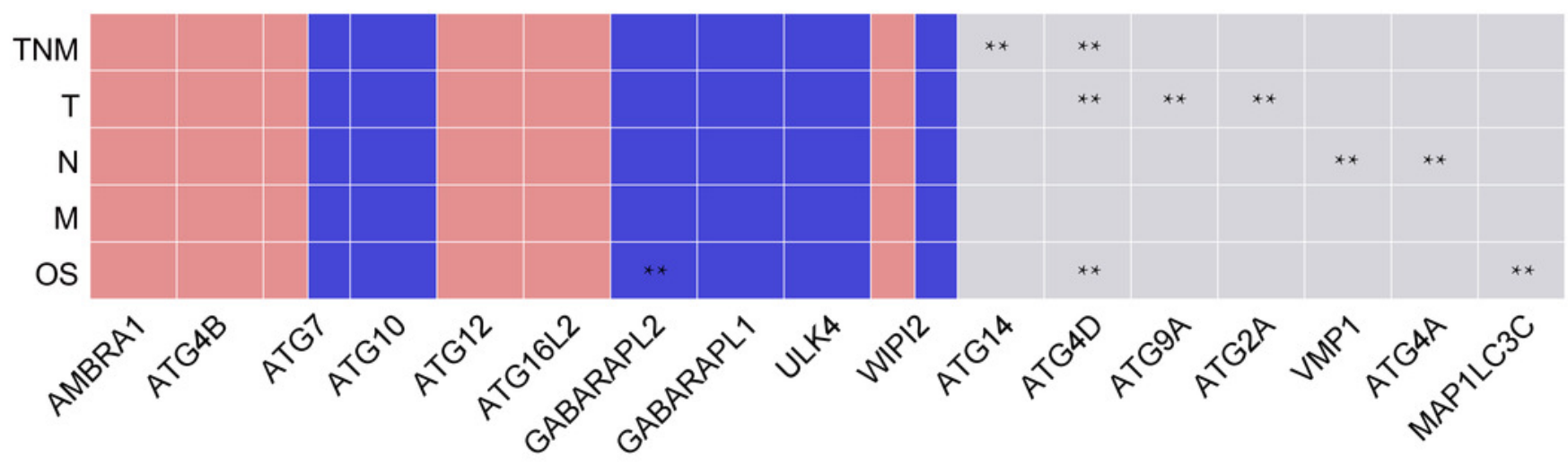


Figure 6

Joint predictive the patients prognosis using ATG4D and MAP1LC3C.

(A)The Kaplan-Meier plot for the four groups. (B)The nomogram for indicating the one- and three-year overall survival in patients with gastric cancer. (C)The calibration curve of nomogram for predicting overall survival at one year. (D)The calibration curve of nomogram for predicting overall survival at three year.

A
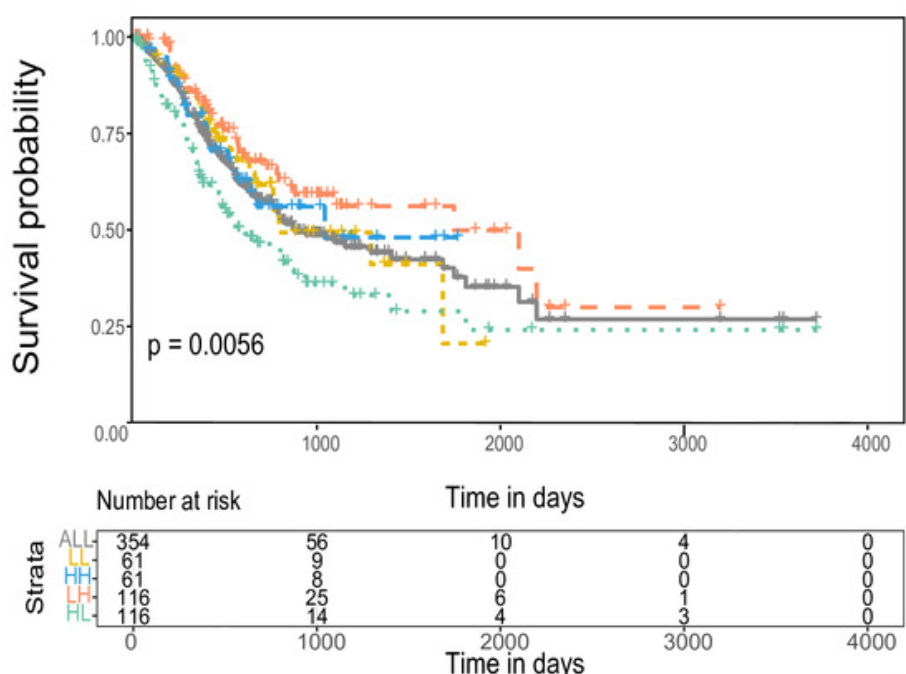

C

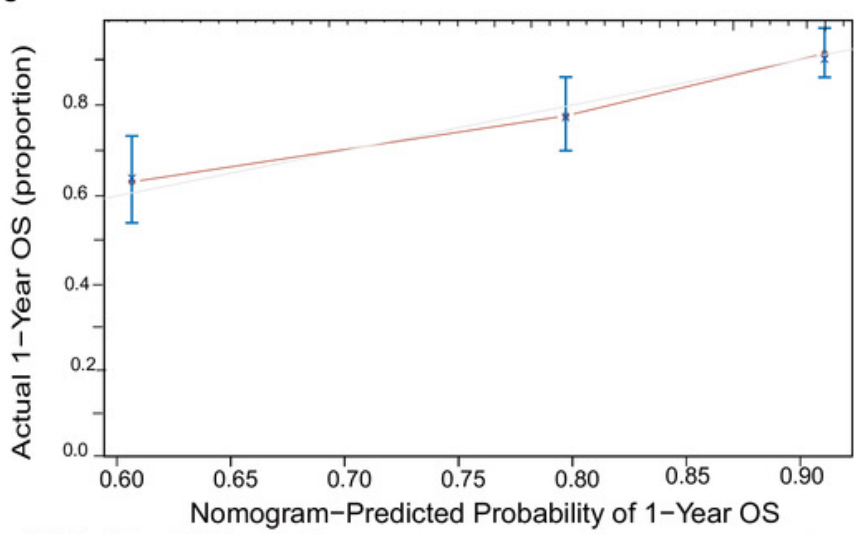

$n=339 d=136 p=6,113$ subjects per group $\quad X-$ resampling optimism added, $B=350$ Gray: ideal
B
Points

ATG

age

gender

stage

Total Points

1-year Survival Probability

3-year Survival Probability
Linear Predictor

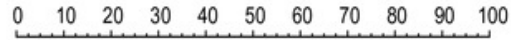

$\overbrace{\mathrm{HL}}^{\mathrm{LL}} \mathrm{HH}$
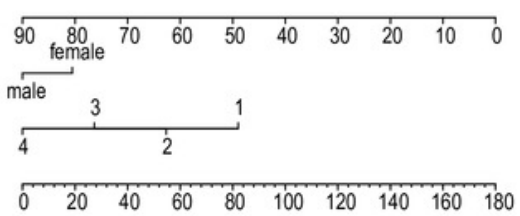

$\begin{array}{llllllllllllllll}4.5 & 5 & 5.5 & 1 & 1 & 1 & 1 & 1 & 1 & 1 & 1 & 1 & 1 & 1 & 1 & 1\end{array}$

$0.20 .30 .40 .50 .60 .70 .8 \quad 0.9$

$\begin{array}{lllll}0.1 & 0.2 & 0.30 .40 .50 .60 .7 & 0.8 & 0.9\end{array}$
D

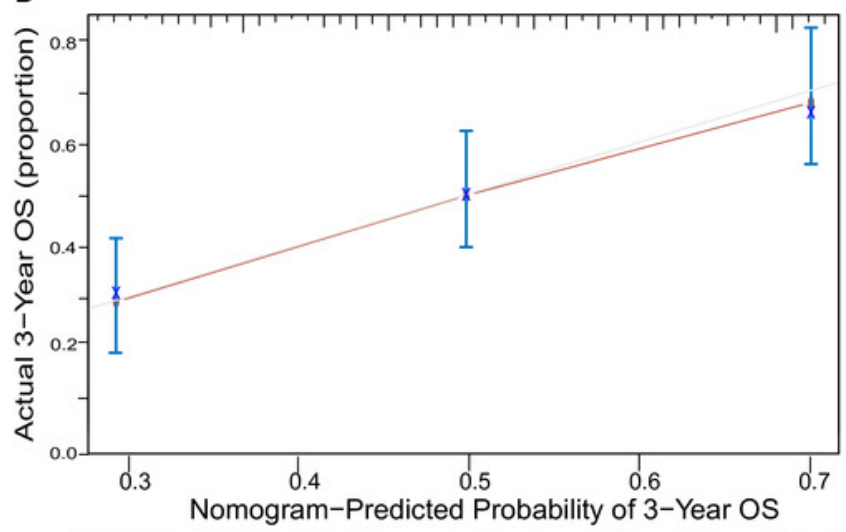

$n=339 d=136 p=6,113$ subjects per group $X-$ resampling optimism added, $B=350$ Gray: ideal 
Table $\mathbf{1}$ (on next page)

Description of autophagy related gene 


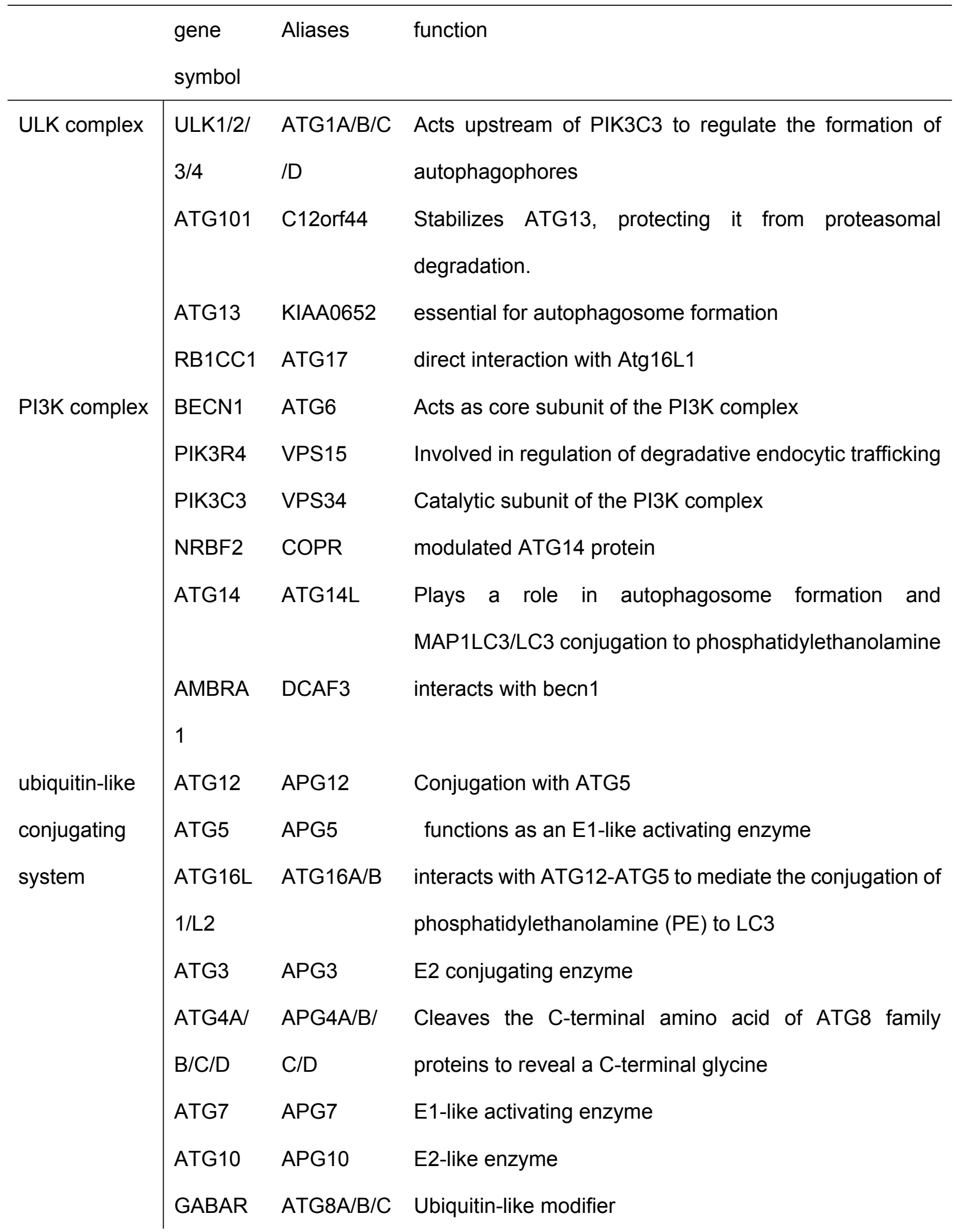


AP/L1/L /D

2/L3

MAP1LC ATG8E/F/G Ubiquitin-like modifier

$3 \mathrm{~A} / \mathrm{B} / \mathrm{B} 2 / \quad / \mathrm{J}$

C

others

WIPI1/2 ATG18A/B Functions upstream of the ATG12-ATG5-ATG16L1 complex and LC3, and downstream of the ULK1 and PI3kinase complexes

ATG9A/ APG9L1/L2 transmembrane protein

B

ATG2A/ I Required for both autophagosome formation

B

ZFYVE1 DFCP1 PI3P-binding FYVE-containing protein

VMP1 EPG3, Plays a role in the initial stages of the autophagic process

TANGO5, through its interaction with BECN1

TMEM49

2 
Table 2 (on next page)

The association between autophagy related gene and TNM stage 


\begin{tabular}{|c|c|c|c|c|c|c|c|}
\hline \multirow[t]{3}{*}{ Gene symbol } & & \multicolumn{3}{|c|}{ TCGA } & \multicolumn{3}{|c|}{ GSE62254 } \\
\hline & & \multicolumn{6}{|c|}{ TNM } \\
\hline & & $|-| \mid$ & III-IV & $P$ & I-II & III-IV & $P$ \\
\hline \multirow{2}{*}{ ATG14 } & low & 96 & 80 & & 72 & 76 & \\
\hline & high & 71 & 105 & 0.00761 & 54 & 96 & 0.02711 \\
\hline \multirow{2}{*}{ ULK3 } & low & 71 & 100 & & 61 & 89 & \\
\hline & high & 96 & 85 & 0.03 & 65 & 83 & 0.568 \\
\hline \multirow{2}{*}{ ATG2B } & low & 95 & 85 & & 62 & 86 & \\
\hline & high & 72 & 100 & 0.04032 & 64 & 86 & 0.8923 \\
\hline \multirow{2}{*}{ ATG12 } & low & 96 & 83 & & 58 & 91 & \\
\hline & high & 71 & 102 & 0.018 & 68 & 81 & 0.241 \\
\hline \multirow{2}{*}{ ATG4C } & low & 94 & 84 & & 65 & 83 & \\
\hline & high & 73 & 101 & 0.041 & 61 & 89 & 0.57 \\
\hline \multirow{2}{*}{ ATG4D } & low & 70 & 100 & & 55 & 95 & \\
\hline & high & 97 & 85 & 0.022 & 71 & 77 & 0.04822 \\
\hline \multirow{4}{*}{ MAP1LC3A } & low & 71 & 105 & & 65 & 83 & \\
\hline & high & 96 & 80 & 0.0076 & 61 & 89 & 0.57 \\
\hline & & \multicolumn{6}{|c|}{$\mathbf{T}$} \\
\hline & & T1T2 & T3T4 & $P$ & T1T2 & T3T4 & $P$ \\
\hline \multirow{2}{*}{ WIPI1 } & low & 41 & 147 & & 101 & 49 & \\
\hline & high & 58 & 121 & 0.022 & 87 & 63 & 0.0947 \\
\hline \multirow{2}{*}{ ATG9A } & low & 39 & 143 & & 80 & 70 & \\
\hline & high & 60 & 125 & 0.018 & 108 & 42 & 0.00083 \\
\hline \multirow{2}{*}{ ATG2B } & low & 42 & 145 & & 95 & 85 & \\
\hline & high & 57 & 123 & 0.047 & 93 & 57 & 0.092 \\
\hline ATG2A & low & 40 & 141 & & 82 & 68 & \\
\hline
\end{tabular}




\begin{tabular}{|c|c|c|c|c|c|c|c|}
\hline & high & 59 & 127 & 0.038 & 106 & 44 & 0.00417 \\
\hline \multirow{2}{*}{ ATG4D } & low & 39 & 141 & & 83 & 67 & \\
\hline & high & 60 & 127 & 0.025 & 105 & 45 & 0.00864 \\
\hline \multirow{4}{*}{ ATG7 } & low & 40 & 146 & & 90 & 60 & \\
\hline & high & 59 & 122 & 0.017 & 98 & 52 & 0.3396 \\
\hline & & \multicolumn{6}{|c|}{$\mathbf{N}$} \\
\hline & & No & !NO & $\mathrm{P}$ & No & !N0 & $\mathrm{P}$ \\
\hline \multirow{2}{*}{ PIK3R4 } & low & 66 & 116 & & 18 & 132 & \\
\hline & high & 45 & 130 & 0.031 & 20 & 130 & 0.728 \\
\hline \multirow{2}{*}{ VMP1 } & low & 65 & 116 & & 28 & 122 & \\
\hline & high & 46 & 130 & 0.046 & 10 & 140 & 0.00178 \\
\hline \multirow{2}{*}{ ATG12 } & low & 66 & 113 & & 16 & 134 & \\
\hline & high & 45 & 133 & 0.018 & 22 & 128 & 0.2976 \\
\hline \multirow{4}{*}{ ATG4A } & low & 68 & 115 & & 26 & 124 & \\
\hline & high & 43 & 131 & 0.0111 & 12 & 138 & 0.01509 \\
\hline & & \multicolumn{6}{|c|}{$\mathbf{M}$} \\
\hline & & M0 & M1 & $\mathrm{P}$ & MO & M1 & $\mathrm{P}$ \\
\hline \multirow{2}{*}{ ULK4 } & low & 160 & 19 & & 137 & 13 & \\
\hline & high & 170 & 6 & 0.008 & 136 & 14 & 0.8401 \\
\hline \multirow{2}{*}{ MAP1LC3B } & low & 174 & 7 & & 139 & 11 & \\
\hline & high & 156 & 18 & 0.0171 & 134 & 16 & 0.3131 \\
\hline
\end{tabular}


Table 3 (on next page)

Prognosis analysis of autophagy related gene in TCGA datasets 
1

\begin{tabular}{ccccc}
\hline & \multicolumn{2}{c}{ Univirable analysis } & \multicolumn{2}{c}{ Multivanable analysis } \\
& $\mathrm{HR}(95 \mathrm{Cl})$ & $P$ & $\mathrm{HR}(95 \mathrm{Cl})$ & $P$ \\
\hline ATG4D & $1.602(1.153-2.225)$ & $\mathbf{0 . 0 0 4 9 3}$ & $1.5727(1.1194-2.21)$ & $\mathbf{0 . 0 0 9 0 5 8}$ \\
GABARAPL2 & $0.6925(0.499-0.9609)$ & $\mathbf{0 . 0 2 7 9}$ & $0.7855(0.5597-1.102)$ & 0.162447 \\
MAP1LC3C & $0.6242(0.4488-0.8682)$ & $\mathbf{0 . 0 0 5 1 1}$ & $0.5767(0.4086-0.8138)$ & $\mathbf{0 . 0 0 1 7 3}$ \\
\hline
\end{tabular}

2 